\title{
The effect of spatial orientation on the perception of moving tactile stimuli
}

\author{
MARTHA A. RINKER and JAMES C. CRAIG \\ Indiana University, Bloomington, Indiana
}

\begin{abstract}
Previous studies have shown that the perception of spatial patterns, such as letters, presented to the hand is affected by the spatial orientation of the hand. The present study investigated how the perception of direction of motion across the fingerpads changes with the position of the hand in space. The moving stimuli were generated on two displays. In one condition, the displays were placed horizontally in front of the subject, with the subject's thumb (target site) and index finger (nontarget site) placed flat on the displays. In a second condition, the displays were vertically oriented and gripped between the thumb and index finger. Using a selective-attention paradigm in which subjects are instructed to respond only to the direction of motion at the target site, performance was still affected by the direction of motion at the nontarget site. Changing the orientation of the displays changed the effectiveness of the nontarget in interfering with the identification of the target movement. Nontarget stimuli that produced no interference in the horizontal orientation did so in the vertical, and vice versa. It appears that subjects are not using the local direction of movement across the fingerpads to judge the relative direction of movement at the two sites; rather, they are using the external direction of movement.
\end{abstract}

Most subjects will respond that they perceive the letter " $d$ " when the lowercase letter " $b$ " is drawn on their foreheads (Duke, 1966). Most subjects will respond " $b$ " when the same letter " $b$ " is drawn on the back of the head. This observation suggests that the perception of tactile spatial patterns is jointly dependent on the local pattern of stimulation on the skin and some kind of spatial coordinate system that is used to interpret the pattern of stimulation. In broad terms, the perception of tactile patterns reflects both bottom-up and top-down processing. A number of ingenious studies have extended this initial observation and demonstrated how altering the position of the stimulated body part in space, the hand, for example, alters the perception of the pattern (Corcoran, 1977; Oldfield \& Phillips, 1983; Parsons \& Shimojo, 1987; Sekiyama, 1991).

In studies by Oldfield and Phillips (1983), Parsons and Shimojo (1987), and Sekiyama (1991), subjects were presented with normal and mirror-reversed letters and numbers at various body sites, including the hand, and were asked to respond whether they perceived each stimulus as being normal or mirror reversed. As the orientation of the hand was varied, the subjects' perceptions of whether the stimuli were normal or mirror reversed were found to vary. Exactly how and under what circumstances the perception of spatial patterns is altered by

This research was supported by Grant DC00095 from the National Institutes of Health. The authors thank Roger P. Rhodes for his assistance in conducting these experiments and Paul M. Evans for helpful comments and suggestions. Correspondence should be addressed to M. A. Rinker, Department of Psychology, Indiana University, Bloomington, IN 47405. spatial orientation is a complicated issue. One complexity is that some subjects may give responses opposite to those given by the majority of subjects. It is speculated that these subjects respond in the way they do because they may be adopting the experimenter's perspective and trying to decide what pattern the experimenter intended to present. Put another way, subjects' responses may be influenced by what they perceive to be the task requirements of the experiment.

Although it is clear that spatial orientation may affect the perception of spatial patterns, it is also clear that in many tasks the spatial orientation of an object is unimportant, and responses should be based exclusively on the local pattern of stimulation. In determining whether the lid on a small jar is loose or in detecting whether a relatively smooth surface is scratched, the orientation of the hand in space is irrelevant. In determining that an object is a key, spatial orientation may also be irrelevant, but in using the key to open a door, the placement of the key in the hand and the orientation of the hand in space is important.

The present study was undertaken to examine the effect of spatial orientation on pattern identification under conditions in which task requirements should play little role in determining the effect of orientation. The present study used a selective-attention task in which a moving target stimulus was presented to the thumb and a nontarget stimulus was presented to the index finger. Previous selective-attention studies had established that when the nontarget moved in a direction different from that of the target it interfered with target identification (Evans \& Craig, 1991). In this selective-attention task, subjects received instructions and training on responding to stim- 
uli presented to the target site but received no instructions for responding to stimuli presented to the nontarget site. Indeed, and critical to the focus of the present study, the subjects were instructed to ignore stimulation presented to the nontarget site.

In these previous studies, the two arrays were placed flat in front of the subject, and there was little question about what constituted same or different directions of movement. When both stimuli moved to the right or both stimuli moved to the left, they were the same (compatible trial). When one stimulus moved to the right and the other moved to the left, they were different (incompatible trial). "Compatible" was defined in terms of the "local" direction of movement, that is, in terms of the sequential activation of receptors across the sensory sheath of the fingerpad. Stimuli presented to two different fingerpads move in the same direction locally if the sequence of activation, for example from right to left, is the same on both fingerpads. In addition, the direction of movement generated on the two displays is similar to the direction of movement that would be encountered if a flat surface were moving below the fingers from left to right or from right to left. Thus, the direction of movement could also be defined by the external direction of movement through space, that is, external to the subject. External direction of movement is defined in terms of a flat surface moving tangentially across the fingerpads. Stimuli presented to two different fingerpads move in the same direction externally if a single flat surface could produce the same pattern of stimulation at the two fingerpads. An external definition of movement is clearly related to Gibsonian notions of object perception and haptic exploration of the environment.

When the two displays are horizontal and side by side, the two ways of defining the direction of movement, local and external, yield the same way of categorizing the pairs of patterns as either compatible or incompatible. What would happen if the two displays were placed back to back, and oriented vertically rather than hori-
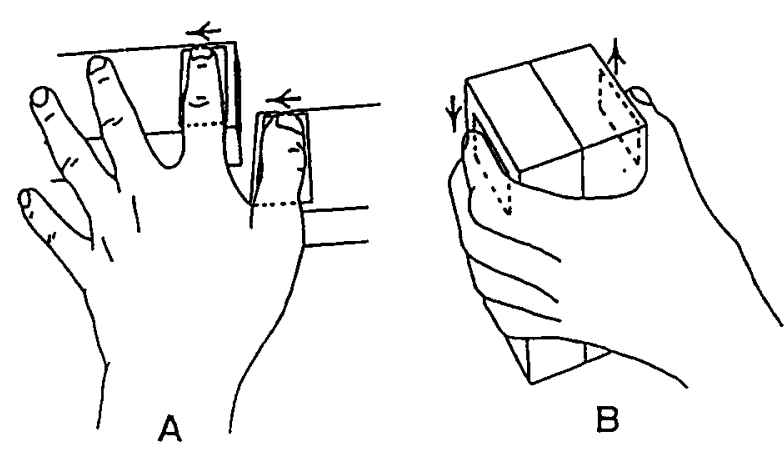

Figure 1. Illustration of display position in the horizontal (A) and vertical (B) orientations. The thumb and index finger are in contact with the active elements of the displays. Arrows indicate the direction of motion across the displays. Note what happens when the same sequential activation across the display horizontally was presented when the displays were oriented vertically.
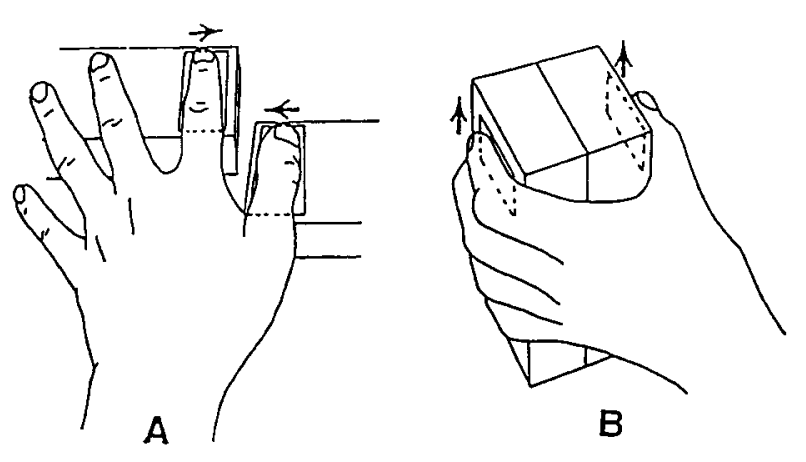

Figure 2. Illustration of display position in the horizontal (A) and vertical (B) orientations. As in Figure 1, arrows indicate the direction of motion across the display.

zontally? Would this change in orientation affect what constitutes an incompatible stimulus? That is, which direction of motion at the nontarget site would interfere with the identification of the target? Figure 1 presents a drawing showing the horizontal and vertical orientations of the two displays. Consider the case when the displays are oriented horizontally and the local directions of movement on both displays are to the left. The external directions are also to the left, as though a surface were passing to the left beneath the fingerpads. When the two displays are placed vertically, the local directions of movement across the two fingerpads remain the same as they were in the horizontal orientation. However, these two local directions produce different external directions, that is, up on the thumb and down on the index finger.

Now consider what happens when the local directions of movement are in the opposite direction for the horizontal displays (Figure 2). These local movements in opposite directions produce externally defined movements in the same direction when the displays are oriented vertically: In this case, it is as though a surface were moving upward between the thumb and index finger.

The present study consists of two experiments. In Experiment 1 , two directions of movement were used to examine the interference effect when the thumb was the target site. The spatial orientation of the two displays was altered, and the nature of the interaction between the target and nontarget sites was measured. In Experiment 2, four directions of movement were tested. Using a paradigm similar to that used by Eriksen and Hoffman (1973) with visual stimuli and Evans and Craig (1992) with tactile stimuli, Experiment 2 examined the level of processing at which the interference between target and nontarget stimuli occurs. This paradigm will be discussed in Experiment 2.

\section{EXPERIMENT 1}

In Experiment 1 only two directions of movement were tested. This simplified the analysis of the results. If target-identification performance is affected by the direction of movement at the nontarget site, it is relatively 
simple to determine the relationship between the directions of movement at the two sites that lead to interference.

\begin{abstract}
Method
Subjects. Nine subjects participated in Experiment 1. Seven were female and two were male. They were drawn from a pool of undergraduate students at Indiana University who were paid employees of the laboratory. All had had previous experience in identifying patterns on the Optacon array. All subjects were right-
\end{abstract} handed.

Apparatus. The apparatus consisted of two tactile arrays interfaced to a PDP-11/34 computer. Each tactile array was identical to that used in the Optacon (Telesensory, Inc.), and each consisted of 144 pins arranged in a rectangular array measuring $11 \mathrm{~mm}$ wide and $27 \mathrm{~mm}$ high. On each array there were 6 columns and 24 rows of pins. Adjacent columns were separated by $2.3 \mathrm{~mm}$, and adjacent rows were separated by $1.2 \mathrm{~mm}$. When activated, the pins vibrated at a frequency of approximately $230 \mathrm{pps}$. Each pin could be activated for durations that were multiples of $4.3 \mathrm{msec}$. The computer controlled the signals to the tactile arrays, the presentation routine, and the visual monitor that was used for instructions and feedback. It also collected the subjects' responses and response latencies. More detailed information about the apparatus can be found in Craig (1980) and Evans and Craig (1991).

Stimuli. The stimuli were presented to the subject's left hand. Movement on each fingerpad was simulated by shifting the locations of activated pins in a column-by-column sequence. For example, to simulate movement across each fingerpad from left to right (radial direction), column 1 of the tactile display was activated for $8.7 \mathrm{msec}$, column 2 was then activated for $8.7 \mathrm{msec}$, and so on, up to column 6 . The total stimulus duration was $52 \mathrm{msec}$. The bottom 18 pins in each column were activated. To simulate movement across each fingerpad from right to left (ulnar direction), column 6 of the tactile display was activated for $8.7 \mathrm{msec}$, followed by the activation of column 5 , and so on across to column 1 . The motion across the arrays was identical in both the horizontal and the vertical orientations. The sequential activation of pins in the Optacon array in this manner leads to a perception of continuous motion across the fingerpad (Evans \& Craig, 1991; Gardner, 1988). As in previous studies, the two arrays were balanced for perceived intensity. Further details about the stimuli may be found in Evans and Craig (1991).

Procedure. Each subject was tested with left arm extended and with the left index fingerpad contacting one tactile array and the left thumb contacting the second array. The subject responded with the index and middle fingers of the right hand, which was resting on a standard keyboard. Two different display orientations, horizontal and vertical, were used. In both orientations, the generation and presentation of stimuli was identical.

The subject was told that tactile patterns would be presented to his/her left index fingerpad and left thumb, and that the patterns would appear to be moving. The subject was instructed to focus attention on the thumb and to report the direction of motion at that site. The subject was instructed to respond by pressing response key 1 if the target stimulus movement started at column 1 and went to column 6 on the display, and to respond by pressing response key 2 if the target stimulus movement started at column 6 and went to column 1 on the display. In both display orientations, the same target movement across the array required the same response by the subject. The subject was never instructed as to how to respond to the nontarget stimulus. In fact, he/she was explicitly instructed to ignore the moving stimulation on the left index fingerpad. Accuracy was stressed, although the subject was asked to respond as quickly as possible. The subject was informed that response times were being recorded.

The subject initiated a trial by pressing either one of the response buttons. Arrows on the buttons indicated the direction of motion, to the left or to the right. These arrows remained unchanged as the orientation of the displays was changed. On every trial, a cuing stimulus, two active pins, was presented at the distal end of both the finger and the thumb for $52 \mathrm{msec}$. The subject was to make sure that he/she could feel both pins on each finger and thus be certain that his/her fingers were placed correctly on both arrays. The subject pressed a third button on the keyboard to indicate that he/she felt the cuing stimuli on both arrays. The subject then pressed either one of the two movement-response keys to continue the trial. One second later, a moving pattern was presented to the left index fingerpad, the nontarget site. The target was presented at either the same time as the nontarget or followed the nontarget presentation by $500 \mathrm{msec}$, a stimulus onset asynchrony (SOA) of $500 \mathrm{msec}$. Following a correct response, the word CORRECT appeared on the visual display for $500 \mathrm{msec}$. Following an incorrect response, the word WRONG appeared on the visual display for $500 \mathrm{msec}$. The visual display then went blank. By pressing either of the response buttons, the subject could initiate the next trial. To eliminate auditory cues produced by the tactile arrays, subjects wore headphones through which white noise was presented. They also wore earplugs.

Each session consisted of six blocks of 48 trials. A session began with one block of single patterns presented to the target site, the left thumb. In the five remaining blocks, both a nontarget and a target were presented. The SOA was varied on a trial-to-trial basis. Within each session, approximately equal numbers of trials were presented at each SOA value. On half of the trials, the two patterns moved in the same direction; on the other half, the two patterns moved in different directions. Each session lasted approximately $30 \mathrm{~min}$. For 5 subjects, the testing began in the horizontal orientation; for 4 subjects, testing began in the vertical orientation. After each subject had completed four sessions in their initial display orientation, they then completed four sessions in the other display orientation.

\section{Results and Discussion}

Because one group of subjects began the task with the displays in the vertical orientation and the other group began the task with the displays in the horizontal orientation, the data from the two groups were initially examined separately. This examination indicated that both groups showed the same interference effects in both the horizontal and vertical conditions. Since no systematic differences were found between the two groups, data from the two groups were collapsed in all analyses. The top panel of Figure 3 presents the percent correct results from the condition in which both displays were placed horizontally. In each condition there were approximately 240 trials $\times 9$ subjects for a total of 2,160 trials. The two-way analysis of variance (SOA $\times$ trial type) was consistent with the pattern of results shown in the top panel of Figure 3, that is, there was a main effect of SOA $[F(1,8)=27.30, p<.001]$, a main effect of trial type $[F(1,8)=29.91, p<.001]$, and an SOA $\times$ trial type interaction $[F(1,8)=10.24, p<.05]$. Single-pattern performance averaged $94 \%$. These results replicate earlier results obtained on other sites on the hand (Evans \& Craig, 1991, 1992; Evans, Craig, \& Rinker, 1992).

The data from the condition in which the displays were placed back to back and oriented vertically are shown in the bottom panel of Figure 3. Single-pattern performance averaged $92 \%$. The main question Experi- 

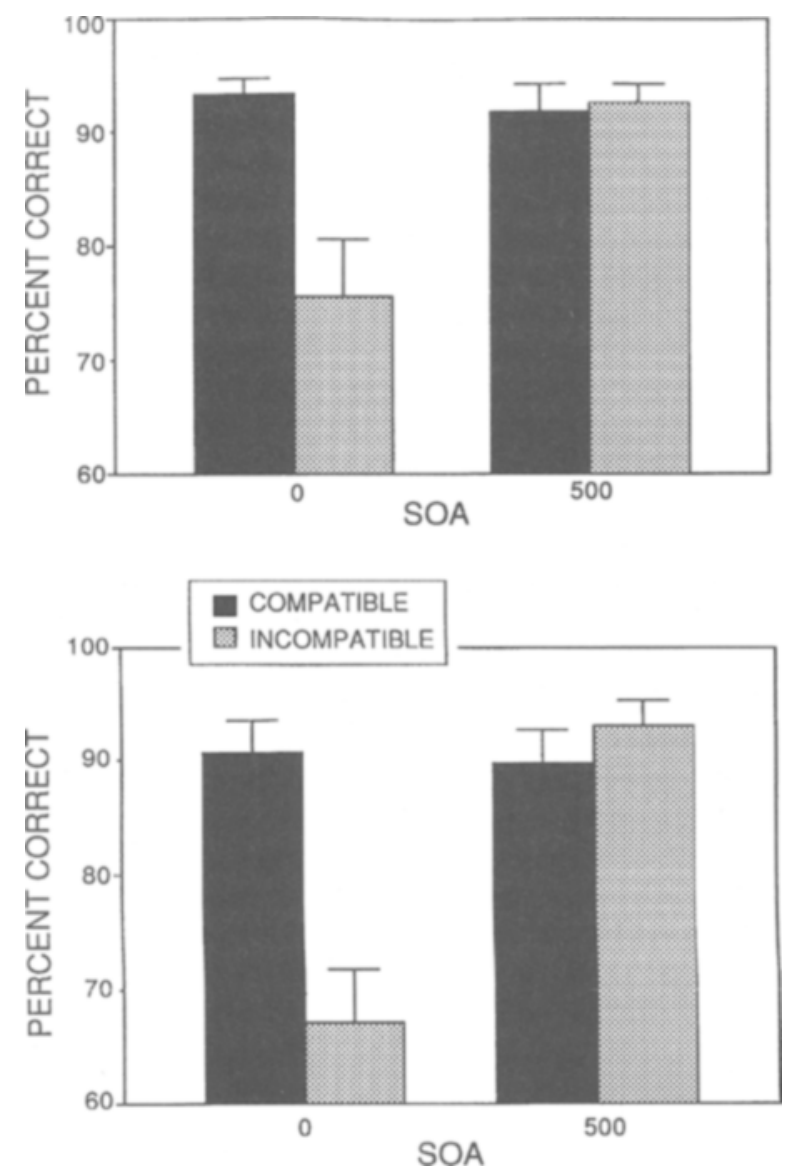

Figure 3. Percent correct target identification for each trial type (compatible and incompatible) as a function of the time in milliseconds between the target stimulus and the nontarget stimulus (SOA). The displays were placed in either the horizontal orientation (top panel) or the vertical orientation (bottom panel). Error bars represent 1 standard error.

ment 1 addressed was whether altering the spatial orientation of the displays would alter the subjects' perception of the direction of movement. The data marked "compatible" refer to those trials on which the external direction of movement was the same at both the target and nontarget locations, and, thus, the local directions of movement differed from one another. The data marked "incompatible" refer to those trials on which the local pattern of motion was the same at the two sites but the external directions of movement differed. The two-way analysis of variance was consistent with the pattern of results shown in the bottom panel of Figure 3 . The analysis showed a main effect of SOA $[F(1,8)=33.78$, $p<.001]$, a main effect of trial type $[F(1,8)=28.12$, $p<.001]$, and an SOA by trial type interaction $[F(1,8)=32.44, p<.001]$.

In terms of the local direction of movement and what constitutes compatible and incompatible, the results in the vertical condition are in direct opposition to the results from the horizontal condition. In terms of the external direction of movement, the results are entirely consistent: No interference is produced when target and nontarget stimuli move in the same external directions, even though the local directions of movement on the fingerpad are opposite to one another. Although the main emphasis of the measurements was on percent correct, the reaction time data were analyzed as well. The pattern of reaction times was consistent with earlier results (Evans \& Craig, 1991, 1992): Reaction times are consistently briefer on compatible than on incompatible trials.

\section{EXPERIMENT 2}

In Experiment 2, a four-stimulus/two-response paradigm (Evans \& Craig, 1992; Eriksen \& Eriksen, 1974) was used to examine the level of processing and the nature of interference between the target and nontarget. Two additional directions of movement, distal-proximal and proximal-distal were used. In this 4-to-2 paradigm, two stimuli are assigned one response and two, another. Previous results (Evans \& Craig, 1992) showed that a physically different nontarget stimulus interfered very little with the perception of the target as long as it was assigned the same response. These results suggest that, for stimuli presented in the horizontal-array position, the nontarget's effect is not due to interference in early stages of processing (masking). Rather, the effect occurs relatively late in processing by the nontarget's activation of a competing response (Evans \& Craig, 1992; Evans et al., 1992). Experiment 2 was designed to examine the extent to which the interference effect observed in Experiment 1 , which was dependent on spatial orientation, was the result of masking or response competition.

\section{Method}

Subjects. Five subjects, drawn from a pool of undergraduate students at Indiana University, participated in Experiment 2. All subjects had had some previous experience at identifying patterns on the Optacon array. Three of these 5 subjects had participated in Experiment 1. All subjects were right-handed.

Apparatus. The apparatus used in Experiment 2 was identical to that used in Experiment 1.

Stimuli. Four directions were used. Two of the directions of movement were the same as those used in Experiment 1, movement across the array from column 1 to column 6 , and vice versa. The remaining two directions of movement, proximal to distal and distal to proximal, were simulated by activating three rows of pins on the display and all six columns simultaneously. To simulate movement in the distal-to-proximal direction, the three rows contacting the distal end of the fingerpad or thumb were turned on for $8.7 \mathrm{msec}$, followed by the next three rows, and so on until the stimulus had swept down all 18 rows in six steps. The total duration, $8.7 \mathrm{msec}$ per step $\times 6$ steps, or $52 \mathrm{msec}$, was identical to the duration of stimuli that moved across the fingerpad. Movement in the proximal-to-distal direction was simulated in a manner opposite to that of the distal-to-proximal direction. Further information about stimulus generation may be found in Evans and Craig (1992).

Procedure. The procedure was identical to that of Experiment 1 except for the following four changes. The first and most important change was the inclusion of two additional directions of motion, proximal to distal and distal to proximal. The subjects were instructed to respond by pressing response key 1 if the tar- 

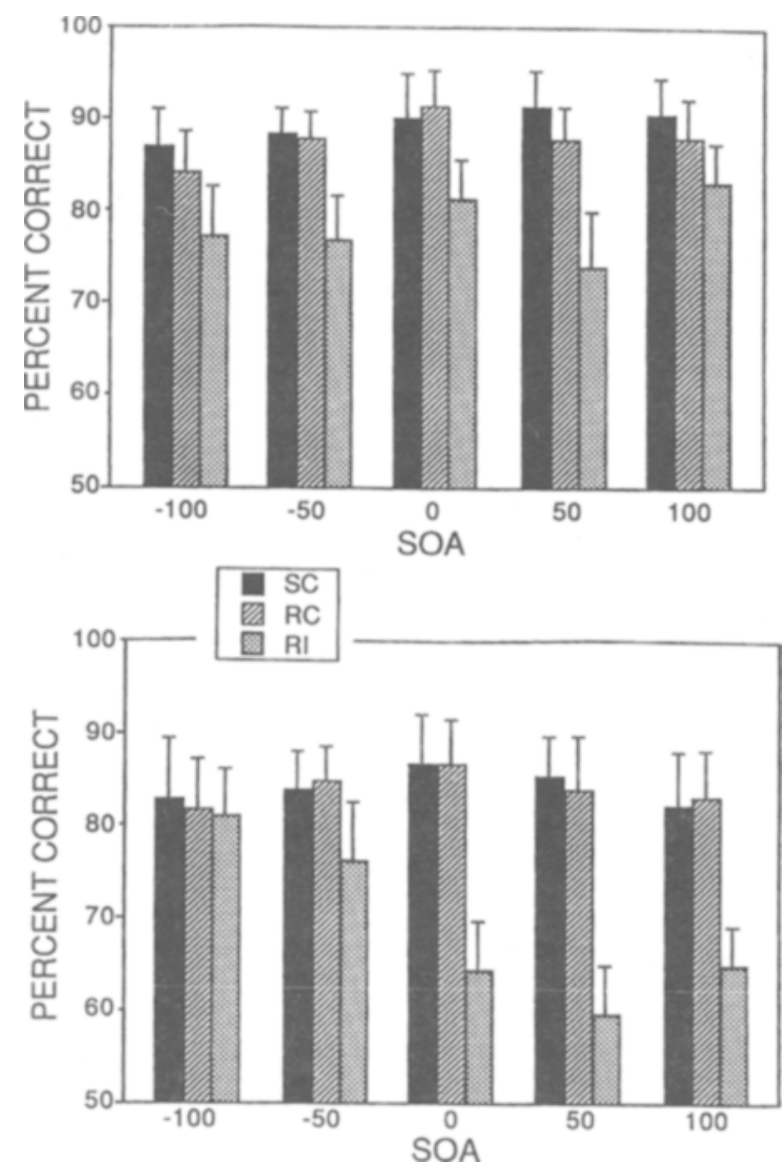

Figure 4. Percent correct target identification for each trial type (SC, $\mathrm{RC}, \mathrm{RI}$ ) as a function of $\mathrm{SOA}$, in milliseconds. Negative SOAs indicate that the nontarget preceded the target. Positive SOAs indicate that the nontarget followed the target. The displays were placed in either the horizontal orientation (top panel) or the vertical orientation (bottom panel). Error bars represent 1 standard error.

get moved from column 6 to column 1 (to the left in the horizontal condition), or from the proximal to the distal end of the thumb. They were instructed to press response key 2 if the target moved from column 1 to column 6 (to the right in the horizontal condition), or from the distal to the proximal end of the thumb.

These four directions of motion, which were mapped to two responses, created three types of trials. On one-fourth of the trials, the two patterns moved in the same direction: stimulus-compatible (SC) trials. On one-fourth of the trials the two patterns moved in different directions but were assigned the same response: responsecompatible (RC) trials. On the remaining trials, the two stimuli moved in different directions and the two directions of movement were assigned different responses: response-incompatible (RI) trials. Comparing performance on the RC trials with performance on RI trials permits us to see the extent to which physically different nontarget stimuli interfere with (mask) targets when they are assigned the same response.

In the second change in procedure from Experiment 1, each session consisted of seven blocks of 50 trials each. As before, each session began with one block of single patterns presented to the left thumb (the target site). The third difference from Experiment 1 was that five SOAs were tested: $-100,-50,0,50$, and $100 \mathrm{msec}$. Negative SOAs refer to trials in which the nontarget preceded the target, positive SOAs to trials in which the nontarget followed the target. The fourth change from Experiment 1 was that testing with all subjects began in the vertical orientation. Each subject completed eight sessions with the vertical display orientation followed by eight sessions with the horizontal orientation.

\section{Results and Discussion}

The top panel of Figure 4 presents the percent correct results when both displays were placed horizontally. In the SC and RC conditions, the data point at each SOA is based on 600 trials; in the RI condition, each data point is based on 1,200 trials. Single-pattern performance, based on 2,000 trials, averaged $93 \%$ correct. A two-way analysis of variance (SOA $\times$ trial type) showed a main effect of trial type $[F(2,8)=25.31, p<.001]$ and an SOA $\times$ trial type interaction $[F(8,32)=3.99, p<.01]$. The relative performance levels on the three trial types are consistent with the results found in previous studies (Evans \& Craig, 1992; Evans et al., 1992). Planned comparisons $(p<.05)$ revealed that at all SOAs performance was equivalent on the SC and RC trials. Nontarget interference was greatest on the RI trials with performance on these trials falling significantly lower than performance on the SC and RC trials at all SOAs. In addition, reaction times were consistent with those of previous studies (Evans \& Craig, 1992; Evans et al., 1992): Reaction times were longer on RI trials than on both $\mathrm{SC}$ and $\mathrm{RC}$ trials.

The results from the vertical orientation are shown in the bottom panel of Figure 4. Single-pattern performance averaged $86 \%$ correct. As in Experiment 1, the definition of the trial types is based on the external, not the local, direction of movement. Table 1 shows how the categorization of trial types for one of the four target stimuli changes when the displays are moved from the horizontal to the vertical orientation. One needs to keep in mind that subjects were instructed to make no judgment of the pattern presented to the nontarget site, that the nontarget patterns contain no information about which target pattern was presented, and that the subjects were instructed to ignore the nontarget pattern. Having said that, the trials were categorized and the data analyzed as though subjects were processing the nontarget patterns. We assumed that subjects were unable to restrict their attention to the target site, perceived the

Table 1

Stimulus-Response Mapping for One Target

\begin{tabular}{lll}
\hline Target & Nontarget & Trial Type \\
\hline & Horizontal & \\
A (left) & A (left) & SC \\
A (left) & B (away) & RC \\
A (left) & C (right) & RI \\
A (left) & D (toward) & RI \\
& Vertical & \\
A (up) & A (down) & RI \\
A (up) & B (away) & RC \\
A (up) & C (up) & SC \\
A (up) & D (toward) & RI \\
\hline
\end{tabular}


movement at the nontarget site, and categorized according to the external direction of movement.

When the displays were in the vertical position, a twoway analysis of variance (SOA $\times$ trial type) revealed a main effect of trial type $[F(2,8)=38.94, p=.0001]$ and an SOA $\times$ trial type interaction $[F(8,32)=4.46, p=$ $.001]$. Planned comparisons $(p<.05)$ showed that performances on SC and RC trials were equivalent over all SOAs. Comparisons of performance on the $\mathrm{SC}$ and $\mathrm{RC}$ trials to that of the RI trials, revealed that performance was significantly lower on the RI trials at the $0-,+50-$, and $+100-$ msec SOAs, but not at the forward SOAs $(-50$ and $-100 \mathrm{msec})$. As found in the horizontal orientation, reaction times were longer on RI trials than on $\mathrm{SC}$ and RC trials.

These results lend support to the view that the interference effect occurs relatively late in processing regardless of the orientation of the hand. The presence of nontarget stimuli that evoked competing responses reduced target identification performance. Changing the orientation of the hand in space had no effect on the amount of masking that the nontarget exerted on the target. More than a physical difference between the target and nontarget is needed to interfere with target identification. To interfere with the target, the direction of movement of the nontarget had to be assigned a response that was incompatible with the response assigned to the target stimulus.

\section{GENERAL DISCUSSION}

The results of the present study are in agreement with those of previous studies that demonstrated that subjects were unable to restrict their attention to a target stimulus presented at one site while ignoring a nontarget stimulus presented at a second site (Evans \& Craig, 1991, 1992; Evans et al., 1992). Also in agreement with previous results, the results from the four-stimuli/tworesponse paradigm in Experiment 2 demonstrated that the interference effect was not due to masking, and suggest that the effect occurs late in processing, presumably at the level of response activation.

The effects of spatial orientation offer additional support for the view that the interference effect occurs during later stages in processing. The fact that altering the orientation of the displays alters the subjects' perception of the relative direction of movement suggests that a given local stimulus can be associated with more than one response. This indicates that the particular responses to the target and nontarget stimuli are evoked after the local directional information and the kinesthetic (orientation) information have been combined, that is, at a later stage of processing.

Consistent with this conceptual view are the findings of a number of neurophysiological studies that used moving stimuli (Costanzo \& Gardner, 1980; Iwamura, Tanaka, Sakamoto, \& Hikosaka, 1983; Warren, Hamalainen, \& Gardner, 1986; Whitsel, Roppolo, \& Werner, 1972). These studies found movement-sensitive neurons in the primary somatosensory projection area (SI) of monkeys. Furthermore, many of the neurons displayed directional sensitivity to stimuli moved across the skin. Gardner (1988) has activated these same neurons with patterns generated on an Optacon display, patterns similar to those used in the present study. Directional sensitivity has been demonstrated even earlier with recordings from peripheral afferents (Goodwin \& Morley, 1987). The presence of such neurons suggests that the local direction of motion is represented at a very early stage of processing. Gardner (1988) reports the presence of some higher order neurons in area 2, labeled "haptic" neurons, which integrate tactile and kinesthetic information from areas $3 \mathrm{~b}$ and 1 . These results indicate that this information is combined somewhat later in processing. The salience of the external direction of movement in the present study may involve similar neurons that combine the local direction of movement at a particular site with kinesthetic information about the orientation of that site. Moreover, to produce the kind of interference seen in Experiments 1 and 2, information about external direction of movement at one site must be compared with movement at another site. This comparison of directions of movement at two sites presumably occurs at even later stages of processing.

Although the way in which subjects use the external direction of movement in the present study appears to be consistent with results from earlier studies in which the perception of a spatial pattern was altered by the position of the hand in space (Corcoran, 1977; Duke, 1966; Oldfield \& Phillips, 1983), the task in the present study differs considerably from the tasks used in the earlier studies. In these earlier studies, letters or numbers were drawn on the subject's palm (Corcoran, 1977) or single letters or numbers were presented to the fingers or palm (Oldfield \& Phillips, 1983; Parsons \& Shimojo, 1987; Sekiyama, 1991). The orientation of the stimulated skin site was varied. The subject judged whether the stimulus pattern corresponded to a stored representation of the letter and then decided if the pattern was presented in a normal or reversed orientation.

In the present study, subjects were required to categorize a single stimulus and explicitly instructed to ignore the nontarget stimuli, so they, presumably, did not compare the target and nontarget stimuli. The task demands should not suggest to the subject how to respond to the nontarget. Moreover, although reaction times in the previously cited studies were not reported, several seconds were probably required to respond. In the present studies, reaction times were much briefer, averaging $500 \mathrm{msec}$ or less. The relatively short reaction times suggest that subjects were more or less automatically combining the local pattern of movement and orientation in space.

The reaction times and the apparent ease with which external, not local, direction of motion is used suggests that the external direction of motion is more salient than other ways in which movement might be encoded. It appears that the movement information that the subject ini- 
tially accesses is the external direction. This is not to say that subjects would be unable to report the local direction of motion, only that it may require more time to do so. Judging local movement may require analyzing the external movement relative to the orientation of the stimulated site. If this were the case, it would be expected that subjects would require more time to indicate the local direction than to indicate the external direction of movement.

\section{REFERENCES}

Corcoran, D. W. (1977). The phenomena of the disembodied eye or is it a matter of personal geography? Perception, 6, 247-253.

Costanzo, R. M., \& GARDNER, E. P. (1980). A quantitative analysis of responses of direction-sensitive neurons in the somatosensory cortex of alert monkeys. Journal of Neurophysiology, 43, 1319-1341.

CRAIG, J. C. (1980). Modes of vibrotactile pattern generation. Journal of Experimental Psychology: Human Perception \& Performance, $\mathbf{6}$, 151-166.

DuKe, J. D. (1966). Perception of finger drawings upon the body surface. Journal of General Psychology, 75, 305-314.

ERIKSEN, B. A., \& ERIKSEN, C. W. (1974). Effects of noise letters upon the identification of a target letter in a nonsearch task. Perception \& Psychophysics, 16, 143-149.

ERIKSEN, C. W., \& Hoffman, J. E. (1973). The extent of processing noise elements during selective encoding from visual displays. Perception \& Psychophysics, 14, 155-160.

Evans, P. M., \& CRAIG, J. C. (1991). Tactile attention and the perception of moving tactile stimuli. Perception \& Psychophysics, 49, 355-364.

Evans, P. M., \& Craig, J. C. (1992). Response competition: A major source of interference in a tactile identification task. Perception \& Psychophysics, 51, 199-206.
Evans, P. M., Craig, J. C., \& Rinker, M. A. (1992). Perceptual processing of adjacent and nonadjacent tactile nontargets. Perception \& Psychophysics, 52, 571-581.

GARDNER, E. P. (1988). Somatosensory cortical mechanisms of feature detection in tactile and kinesthetic discrimination. Canadian Journal of Physiology \& Pharmacology, 66, 439-454.

Goodwin, A. N., \& MorLEY, J. W. (1987). Sinusoidal movement of a grating across the monkey's fingerpad: Effect of contact angle and force of the grating on afferent fiber responses. Journal of Neuroscience, 7, 2192-2202.

Iwamura, Y., Tanaka, M., SaKamoto, M., \& Hikosaka, O. (1983). Converging patterns of finger representation and complex response properties of neurons in area 1 of the first somatosensory cortex of the conscious monkey. Experimental Brain Research, 51, 327-337.

Oldfield, S. R., \& Phillips, J. R. (1983). The spatial characteristics of tactile form perception. Perception, 12, 615-626.

Parsons, L. M., \& Shimojo, S. (1987). Perceived spatial organization of cutaneous patterns on surfaces of the human body in various positions. Journal of Experimental Psychology: Human Perception \& Performance, 13, 488-504.

SekrYAMA, K. (1991). Importance of head axes in perception of cutaneous patterns drawn on vertical body surfaces. Perception \& Psychophysics, 49, 481-492.

Warren, S., Hamalainen, H., \& Gardner, E. P. (1986). Objective classification of motion- and direction-sensitive neurons in primary somatosensory cortex of awake monkeys. Journal of Neurophysiology, 56, 598-622.

Whitsel, B. L., Roppolo, J. R., \& Werner, G. (1972). Cortical information processing of stimulus motion on primate skin. Journal of Neurophysiology, 35, 691-717.

(Manuscript received May 24, 1993: revision accepted for publication March 28, 1994.) 